\title{
Le complexe parasitaire du pied du haricot. I. Mise en évidence des principaux champignons responsables de la maladie : Fusarium solani f. sp. phaseoli et Thielaviopsis basicola
}

Joël LECHAPPÉ, Francis ROUXEL \& Marie-Thérèse SANSON

I.N.R.A., Station de Pathologie végétale, Centre de Recherches de Rennes, B.P. 29, F35650 Le Rheu

RÉSUMÉ

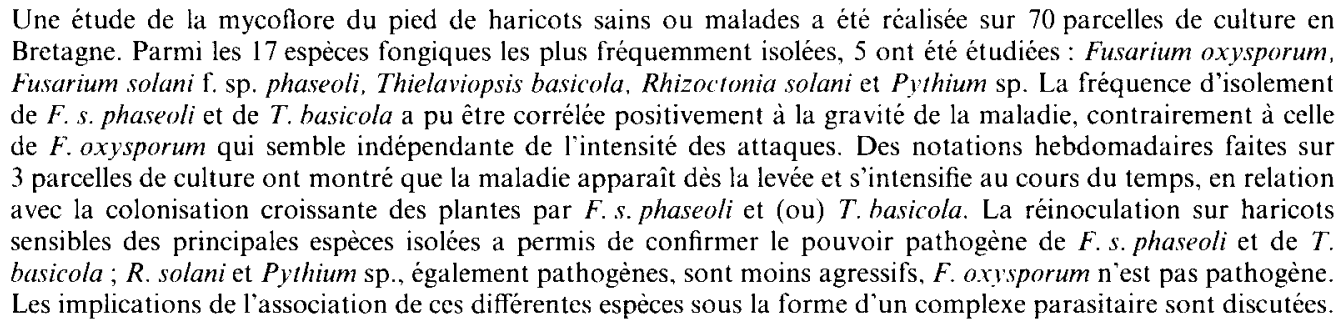

Mots clés additionnels: Phaseolus vulgaris, racines, collet, diagnostic, pouvoir pathogène, Fusarium oxysporum, Rhizoctonia solani, Pythium. Fusarium solani F. sp. phaseoli and Thielaviopsis basicola.

The bean foot mycoflora was investigated on healthy or diseased plants, in 70 fields cultivated in beans in Brittany. Among 17 fungi species isolated from bean foots, five were studied : Fusarium oxysporum, Fusarium solani f. sp. phaseoli, Thielaviopsis basicola, Rhizoctonia solani and Pythium sp. The frequency of occurrence of $F$. s. phaseoli or T. basicola isolation was positively correlated with disease rate. Further weekly observations made in 3 fields showed a disease development on bean seedlings increasing with time, in relation to the increase of plant colonization by F. s. phaseoli and/or T. basicola; pathogenicity of F. s.phaseoli and T. basicola was shown after inoculation. $R$. solani and Pythium sp. were less aggressive than the first two species; F. oxysporum was not pathogenic. The consequences of the association of the 5 species into a parasitic complex are discussed.

Additional key words : Phaseolus vulgaris, root, collar, diagnostic, pathogenicity, Fusarium oxysporum, Rhizoctonia solani, Pythium.

\section{INTRODUCTION}

La maladie du pied du haricot, dont les symptômes visibles à l'arrachage se caractérisent par la présence de nécroses brun-rougeâtres sur les racines et le collet des plantes, est largement répandue dans les zones traditionnelles productrices de haricots verts et de flageolets. Les dégâts qui en résultent se traduisent par des réductions de rendement, des dépréciations de la qualité du produit, et dans les cas les plus graves, par la perte totale de la récolte. Bien qu'il soit difficile d'évaluer préci- sément son incidence, les pertes qu'elle occasionne sont parfois suffisamment importantes pour contraindre certains producteurs à abandonner la culture du haricot, faute de méthode de lutte.

Cette maladie, décrite en 1919 par BURKHOLDER aux Etats-Unis, a été très étudiée depuis. Elle est le plus souvent appelée « Nécroses de racines et pourritures du collet 》 (Messiaen \& Lafon, 1970), "Complexe parasitaire des racines du Haricot " (DAVET et al., 1980) ou "Maladie du pied", ce qui correspond au "Root rot 
complex », « Dry root rot », ou plus simplement « Root rot " des chercheurs anglo-saxons. Si les différents auteurs s'accordent assez bien sur la terminologie à employer, comme sur l'origine parasitaire de la maladie, par contre des divergences apparaissent lorsqu'il s'agit de préciser l'identité du ou des parasites en cause, d'où des dénominations parfois plus précises: «Fusarium root rot» (BURKe, 1965), «Pythium root rot» (Pieczarka \& Abawi, 1978).

En fait, la littérature fait état de 5 espèces fongiques pouvant intervenir seules ou en association: en 1919 , BURKHOLDER associe la maladie à la présence de Fusarium solani f. sp. phaseoli, champignon considéré plus tard comme agent primaire par MALOY (1959) et BURKE (1965) aux Etats-Unis, mais également par GUPTA \& SaHARAN (1973) en Inde et Davet et al. (1980) au Liban. Papavizas \& Davey (1961), puis Papavizas \& ADAMS (1969) attribuent par contre ce rôle à Thielaviopsis basicola que beaucoup considèrent plutôt comme un parasite de faiblesse. Pour d'autres auteurs, notamment SirRy et al. (1974) en Egypte, SUMmer et al. (1978) puis CAMPBELl et al. (1980) aux Etats-Unis, les nécroses racinaires du haricot seraient dues à Rhizoctonia solani, tandis que PIECZARKa \& ABAwi (1978) et REELEDER (1981) font état de pourritures dues à Pythium ultimum et Pythium irregulare. Enfin, toujours aux Etats-Unis, PFENDER \& HAGEDORN signalent en 1982 l'apparition d'une nouvelle maladie des racines qu'ils attribuent à Aphanomyces euteiches $\mathrm{f}$. $\mathrm{sp}$. phaseoli.

Cette revue bibliographique rapide montre que si l'existence d'un complexe parasitaire semble généralement admise, il existe en fait une grande diversité de situations selon les auteurs et les régions étudiées, et la plupart des travaux restent limités à l'étude du ou des parasites considérés comme les plus importants.

En France, aucune étude de base n'avait été entreprise sur le sujet jusqu'ici. Les seules données bibliographiques (MESSIAEN \& LAFON, 1970; Decharme \& Coutın, 1980) attribuant un rôle à Fusarium solani f. sp. phaseoli résultent d'observations et d'analyses ponctuelles difficilement généralisables; c'est ce qui dans un premier temps, nous a amenés à inventorier la microflore associée à la manifestation de cette maladie.

L'étude présentée ici a pour but de déterminer le ou les agents responsables de la maladie du pied du haricot en France et plus particulièrement en Bretagne; elle comporte deux volets :

- la recherche de liaisons entre la manifestation de la maladie et la composition de la mycoflore du pied du haricot ;

- l'étude du pouvoir pathogène des principales espèces fongiques mises en évidence.

\section{MATÉRIEL ET MÉTHODES}

\section{A. Recherche de liaisons entre la présence de la maladie et la mycoflore du pied du haricot}

\section{Prélèvements d'échantillons}

L'inventaire de la mycoflore est réalisé sur 70 parcelles de culture réparties sur l'ensemble des zones de produc- tion du haricot en Bretagne (fig. 1). Les échantillons végétaux sont recueillis pendant la période de récolte ( 15 août-30 septembre), à raison de 50 plantes par parcelle prélevées en 10 placettes selon les deux diagonales.

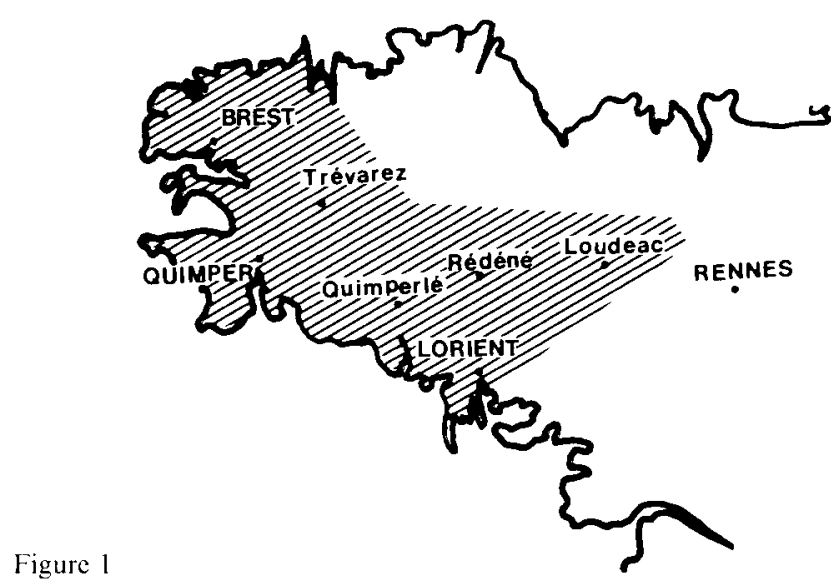

Zones de culture du haricot sur lesquelles 70 parcelles ont fait l'objet d'un suivi régulier de la maladie du pied.

French bean crop areas where foot disease was studied in 70 fields.

L'évolution de la mycoffore est étudiée sur 3 parcelles situées à Rédéné, Quimperlé et Trévarez (fig. 1) ; chaque parcelle fait l'objet d'une prise d'échantillon chaque semaine du semis à la récolte ( 15 juin- 15 septembre) selon le protocole de prélèvement ci-dessus.

\section{Evaluation de la maladie}

Elle est réalisée au laboratoire après lavage et répartition des plantes en 5 classes en fonction de l'importance des symptômes sur le collet et le système racinaire.

L'affectation d'un coefficient à chaque classe $(0-0,25$ 0,5-0,75-1) permet le calcul d'un indice pathologique (IP) qui traduit l'importance de la maladie :

$$
I P=\sum_{i=0}^{i=4} \frac{\mathrm{Ni} \times \mathrm{Ci} \times 100}{\mathrm{~N}}
$$

$$
\begin{aligned}
& \mathrm{Ni}=\text { Nombre de plantes dans la classe } \mathrm{i} \\
& \mathrm{Ci}=\text { Coefficient attribué à la classe } \mathrm{i} \\
& \mathrm{N}=\text { Nombre total de plantes }
\end{aligned}
$$

\section{Analyse de la flore fongique}

Les analyses sont réalisées sur un sous-échantillon de 10 plantes. Celles-ci sont lavées, désinfectées à l'alcool et séchées par un passage rapide à la flamme. La base du collet est ensuite découpée en 10 fragments d'environ $2 \mathrm{~mm}$ qui sont déposés sur un milieu nutritif approprié :

- milieu malt gélosé additionné de sulfate de streptomycine à $50 \mathrm{mg} / \mathrm{l}$ pour l'isolement de la mycoflore classique.

- milieu spécifique (NASH \& SNYDER, 1962) pour lisolement des Fusarium.

Les cultures sont incubées pendant 8 jours à la température ambiante. Dans la semaine qui suit, les colonies qui se sont développées sont identifiées et dénombrées. 
TABLEAU

Identité des 20 isolats éfudiés pour leur agressivité sur haricot et appartenant aux 5 principales espèces fongiques constituant le complexe parasitaire Identity of 20 fungal isolates from the parasitic complex tested for aggressiveness on beans.

\begin{tabular}{|c|c|c|c|}
\hline \multirow{2}{*}{ Espèces } & \multicolumn{2}{|c|}{ Nature de l'isolat } & \multirow{2}{*}{$\begin{array}{c}\text { Date } \\
\text { d'isolement }\end{array}$} \\
\hline & Code & Origine & \\
\hline Fusarium solani f. sp. phaseoli & $\begin{array}{l}\text { FSP } 111 \\
\text { FSP } 105 \\
\text { FSP } 124 \\
\text { FSP } 150\end{array}$ & $\begin{array}{l}\text { TREVAREZ (29) } \\
\text { RIEC/BELON (29) } \\
\text { MOREAC (56) } \\
\text { CORAY (29) }\end{array}$ & $\begin{array}{l}1982 \\
1981 \\
1981 \\
1982\end{array}$ \\
\hline Thielaviopsis hasicola & $\begin{array}{l}\text { TB } 130 \\
\text { TB } 169 \\
\text { TB } 107 \\
\text { TB } 93\end{array}$ & $\begin{array}{l}\text { MOREAC (56) } \\
\text { RIEC/BELON (29) } \\
\text { TREVAREZ (29) } \\
\text { MOREAC }(56)\end{array}$ & $\begin{array}{l}1982 \\
1982 \\
1982 \\
1982\end{array}$ \\
\hline Fusarium oxysporum & $\begin{array}{ll}\text { FO } & 131 \\
\text { FO } & 137 \\
\text { FO } & 172 \\
\text { FO } & 146\end{array}$ & $\begin{array}{l}\text { MOREAC }(56) \\
\text { TREGUNC (29) } \\
\text { NEUILLAC }(22)^{*} \\
\text { TREVAREZ }(29)^{*}\end{array}$ & $\begin{array}{l}1982 \\
1982 \\
1982 \\
1981\end{array}$ \\
\hline Rhizoctonia solani & $\begin{array}{ll}\text { RH } & 32 \\
\text { RH } & 31 \\
\text { RH } & 30 \\
\text { RH } & 33\end{array}$ & $\begin{array}{l}\text { RIEC/BELON (29) } \\
\text { MOELAN/MER (29) } \\
\text { MOELAN/MER (29) } \\
\text { MOREAC }(56)\end{array}$ & $\begin{array}{l}1981 \\
1981 \\
1981 \\
1982\end{array}$ \\
\hline Pythium sp. & 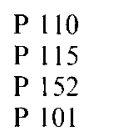 & $\begin{array}{l}\text { CORAY (29) } \\
\text { CORAY (29) } \\
\text { SCAER (29) } \\
\text { RIEC/BELON (29) }\end{array}$ & $\begin{array}{l}1981 \\
1981 \\
1982 \\
1981\end{array}$ \\
\hline
\end{tabular}

Toutes les souches sont isolées de haricots malades, sauf deux $\left({ }^{*}\right)$ isolées de haricots sains.

Strains isolated from attacked beans, except for two $\left({ }^{*}\right)$ isolated from healthy beans.

Compte tenu du manque de fiabilité des techniques d'isolement du $T$. basicola, ce champignon a été recherché par examen direct à la loupe binoculaire $(\times 50)$ des fragments de collets de plantes déposés en boîtes de Petri.

Les résultats sont exprimés soit en pourcentage de parcelles contaminées, soit en pourcentage de plantes colonisées, soit enfin en pourcentage de fragments colonisés par échantillon (taux de colonisation).

Les fréquences des principales espèces fongiques dénombrées sont ensuite comparées aux indices pathologiques mesurés dans les parcelles correspondantes.

\section{B. Etude du pouvoir pathogène des principales espèces fongiques mises en évidence}

\section{Matériel étudié}

L'étude a porté sur 20 isolats appartenant à 5 espèces (tabl. 1). Ces isolats proviennent de haricots malades à l'exception de 2 prélevés sur haricots sains.

\section{Méthodes d'infestation artificielle}

Deux techniques ont été mises en cuvre:

- L'inoculum de Fusarium solani f. sp. phaseoli, Fusarium oxysporum, Rhizoctonia solani et Pythium sp., est produit sur vermiculite imbibée par un demi-volume d'eau contenant 2 p. 100 d'extrait de malt; la vermiculite, conditionnée dans des sacs en polyéthylène résistant à l'autoclavage, est d'abord autoclavée seule $\left(120^{\circ} \mathrm{C}\right.$ $60 \mathrm{~min}$ ), puis après addition de la solution maltée $\left(120^{\circ} \mathrm{C}-20 \mathrm{~min}\right)$. Après ensemencement du champignon sous forme d'explantat, la culture est incubée pendant 15 jours à $25^{\circ} \mathrm{C}$.

En fin d'incubation, le contenu des sacs est incorporé au substrat de culture, un mélange terreux $(1 / 3$ terre franche, $1 / 3$ sable, $1 / 3$ tourbe) préalablement traité à la vapeur pendant $1 \mathrm{~h}$, à raison de 1 volume de vermiculite pour 10 volumes de substrat. Le substrat infesté est ensuite réparti en pots de $9 \mathrm{~cm}$ de diamètre, dans chacun desquels on sème 5 graines désinfectées de haricot (var. "GITANA »). L'essai qui comprend 5 répétitions est réalisé sous serre où la température est maintenue entre 20 et $25^{\circ} \mathrm{C}$. La durée de la culture est de 7 semaines.

L'inoculum de Thielaviopsis basicola est produit en fiole d'Erlenmeyer sur une solution maltée à $2 \mathrm{p}$. 100. La culture est incubée à $22{ }^{\circ} \mathrm{C}$ en agitation continue et à l'obscurité. Après 4 jours d'incubation, les spores du champignon sont recueillies par passage sur gaze stérile, centrifugation (3000 tours/min) puis rinçage à l'eau stérile. Cette opération est répétée 3 fois.

La culture de plantes (var. "GITANA ») est réalisée sur perlite en tubes à essais de $22 \mathrm{~mm}$ de diamètre. L'infestation a lieu lorsque les plantules atteignent le stade "crosse"; chaque tube, contenant un individu, reçoit $6 \mathrm{ml}$ d'inoculum sous forme de suspension de spores à raison de $10^{3}$ propagules $/ \mathrm{ml}$ de perlite. Les cultures sont ensuite placées en enceinte climatisée pendant 15 jours à $22{ }^{\circ} \mathrm{C}$, tubes ouverts. Chaque traitement est répété 10 fois.

- Notation: en fin d'essai, les plantes sont arrachées, lavées, puis réparties en 5 classes selon la gravité de l'attaque sur le collet et les racines; l'indice pathologique est ensuite calculé selon la formule présentée précédemment. 


\section{RÉSULTATS}

\section{A. Mise en évidence de liaisons entre la présence de la maladie et la composition de la mycoflore du pied du haricot}

\section{Inventaire du cortège parasitaire du pied du haricot}

Les résultats montrent l'importance relative des différents champignons qui colonisent les pieds de haricots sains et malades confondus (tabl. 2). Les Fuscirium (F. oxysporum, F. solani, F. roseum), constituent la majeure partie des espèces isolées. Ils sont accompagnés d'espèces saprophytes (Trichoderma sp., 16 p. 100 des plantes), Mucorales (15 p. 100), Gliocladium $\mathrm{sp}$. (12,2 p. 100), Aspergillacées (9,5 p. 100). A côté d'elles se trouve tout un cortège de champignons peu fréquents dans nos conditions d'isolement: Rhizoctonia solani, Pythium sp., Thielaviopsis basicola, Geotrichum sp., Cephalosporium sp.

Il apparaît donc que les Fusarium colonisent presque systématiquement les pieds de haricots, quils soient sains ou malades, et que cette colonisation est dense (taux de colonisation total voisin de 81 p. 100).

Parmi les isolats de $F$. solani présents, nous avons distingué 2 types :

- L’un, à mycélium blanc aérien, croissance rapide, correspond à l'espèce solani classique (taux de colonisation : 1 p. 100).

- L'autre, à mycélium rasant bleu-vert, croissance lente, produisant des macroconidies plus longues et plus abondantes, présente les critères de la forme spéciale phaseoli décrite par MESSIAEN \& CASSINı (1968) (taux de colonisation : 18,7 p. 100).

\section{TABLEAU 2}

Inventaire de la flore fongique isolée des pieds de haricot (sams et malades confondus) prélev's dans 70 parcelles de culture.

Analysis of the fungal microftora isolated from attacked and heatlry. beans laken in 70 fields

\begin{tabular}{lcc}
\hline \multicolumn{1}{c}{ Champignons isoles } & $\begin{array}{c}\text { Plantes } \\
\text { colonisces } \\
\%\end{array}$ & $\begin{array}{c}\text { Taux de } \\
\text { colonisation } \\
\%\end{array}$ \\
\hline Alternaria sp. & 2 & 0.15 \\
Aspergillacées & 9,5 & 3,0 \\
Botrytis sp. & 1 & 0,1 \\
Cephalosportum sp. & 1 & 0.2 \\
Cylindrocarpon sp. & 0,01 & 0.01 \\
Epicocim sp. & 0,8 & 0.01 \\
Fusarium oxisportum & 96 & 59.16 \\
Fusarium roseum & 18 & 2.6 \\
Fusarium solani & 3,9 & 1 \\
Fusarium solani f. sp. phaseoli & 32,4 & 18,7 \\
Geotrichum sp. & 1,2 & 0.2 \\
Gliocladium sp. & 12,24 & 3,8 \\
Mucorales & 15 & $*$ \\
Pythium sp. & 1 & $*$ \\
Rhizoctonia solani & 4,1 & $*$ \\
Stemphylium sp. & 1,5 & 0,5 \\
Thielariopsis hasicola & 0,1 & 0,12 \\
Trichoderma sp. & 16 & $*$ \\
Divers & 5,5 & 0,8 \\
& & \\
\hline
\end{tabular}

* Non quantifrable.

* No quantitative analysis.
2. Relations entre la gravité de la maladie et l'abondance de certaines espèces fongiques

La répartition de la maladie dans les 70 parcelles suivies peut être comparée à l'abondance de 5 espèces retenues soit en raison de leur fréquence d'isolement ( $F$. oxysporum et $F . s$, phaseoli), soit parce qu'elles sont considérées comme potentiellement pathogènes sur haricot à partir des données de la littérature ( $P$ ythium sp., R. solani, T. basicola) (fig. 2-3).

- Les F. oxysportum (fig. 2) sont très abondants (96 p. 100 des plantes colonisées) et leur fréquence d'isolement est indépendante de la présence ou non de la maladie; ceci confirme les travaux de TAYLOR \& PARKINSON (1965) qui considèrent les F.oxysporum comme les principaux champignons colonisateurs des racines de haricots sains.

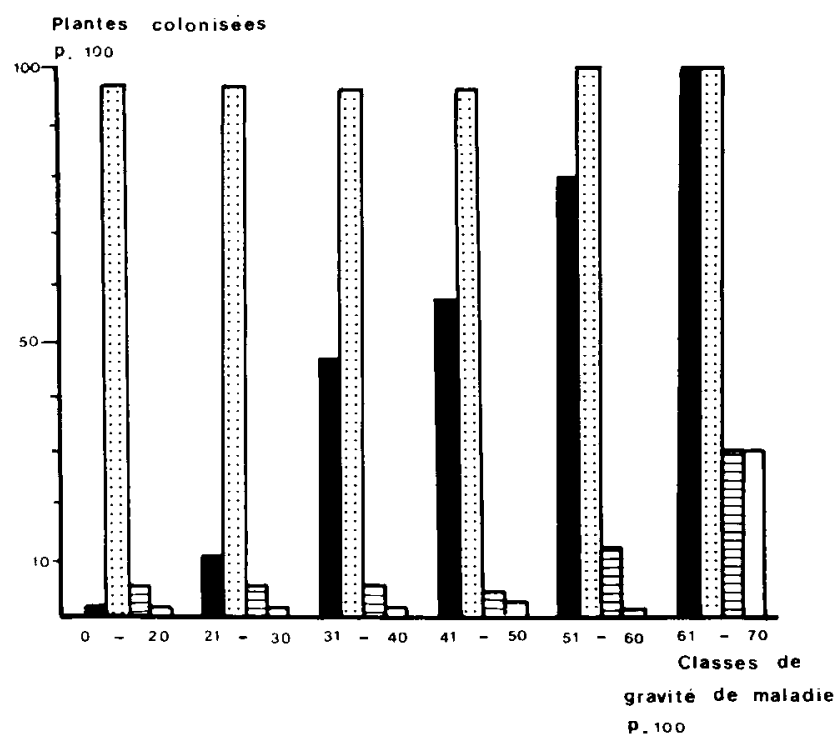

Figure 2

Relations entre la gravite de la maladie du pied du haricot et le pourc'mage de plantes solonisées par les principales espèces fongiaues isolées sur milieu matritif: F.s. phaseoli ( $\mathbf{0})$. F. oxysporum ( $:$ : , R. solani (瞳). Pythium $s p .(7)$

Etude rialiscís sur 70 parcelles.

Relationship between intensity of hean foot disease and percentage of plants colonized by the main fungi specie's isolated on nutrient medium

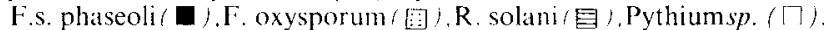
Data from 70 ficlds.

- Le pourcentage de plantes colonisées par F. s. phaseoli est corrélé positivement à la gravité de l'attaque; la même tendance apparait avec $R$. solani, mais à un degré moindre. Les Pythium sp. sont isolés moins fréquemment et seulement dans les parcelles où les attaques sont graves : la colonisation ne dépasse pas 30 p. 100 pour des indices pathologiques compris entre 60 et 70 p. 100 . Un lavage des plantes à l'eau et l'utilisation d'un milicu sélectif à base de bénomyl n'augmentent pas leur fréquence d'isolement.

- L'illustration des observations réalisées sur la fréquence de $T$. basicola montre que ce champignon est fréquent en culture, et que sa présence est corrélée positivement à la gravité de la maladie (fig. 3). Ces 


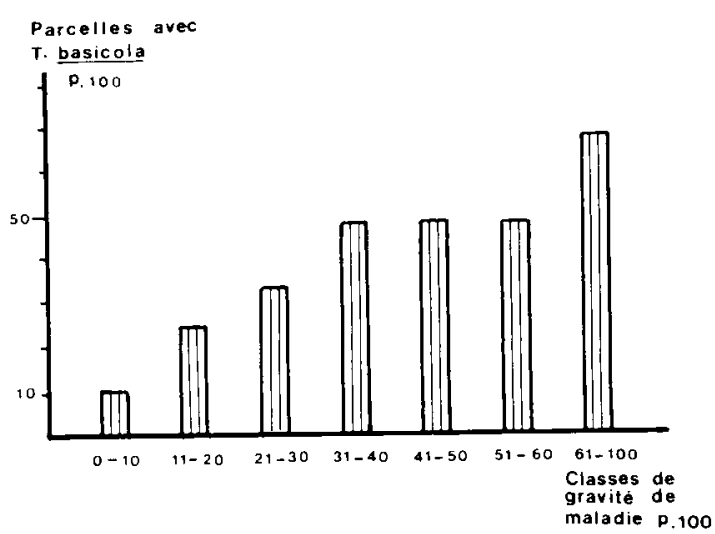

Figure 3

Relations contre la gravite de la maladie du pied dis haricor et te pourcentage de parcelles toucheses par $\mathrm{T}$. basicola ( III ).

Enude réalisée sur 70 parcelles.

Relationship herreen intensity of bean foot disease and oc curence of $\mathrm{T}$. basicola Ull

Data from ru fields.

observations confirment le risque déjà suggéré par DAvet et al. (1980) de sous-estimer la fréquence de T. hasicola par les techniques classiques d'isolement.

3. Relations entre l'évolution de la gravité de la maladie' en cours de culture et la mycoflore associée

Cette étude, réalisée sur 3 parcelles, a porté sur les 3 espèces les plus fréquemment observées: $F$. s. phascoli, F. oxysporum, T. basicola (fig. 4).

\section{a) Parcelle de Trévarez}

Les attaques sont très limitées; les populations de $F$, oxysporum sont élevées et celles de $F$. s. phaseoli très faibles; $T$. hasicola est absent.

\section{b) Parcelle de Quimperle'}

Cette parcelle se différencie de la précédente par un taux d'attaque élevé (46 p. 100 en fin de culture) et la présence de populations importantes de F.s.phaseoli; celui-ci se manifeste très tôt, moins de 15 jours après le semis, et le taux de colonisation évolue corrélativement à l'intensité d'attaque. Le taux de colonisation par F. oxysporum est élevé (entre 28 et 67 p. 100); $T$. basicola est absent.

\section{c) Parcelle de Rédéné}

Le niveau d'attaque est très élevé ; les populations de F. oxysporum sont abondantes; le F. s. phaseoli apparaît tôt après le semis et sa densité augmente progressivement en fonction du temps ; cependant, elle se maintient à un niveau inférieur à celui mesuré dans la parcelle de Quimperlé, pour un taux d'attaque voisin. Il est particulièrement intéressant de noter l'apparition de T. basicola moins de 15 jours après le semis, avant le
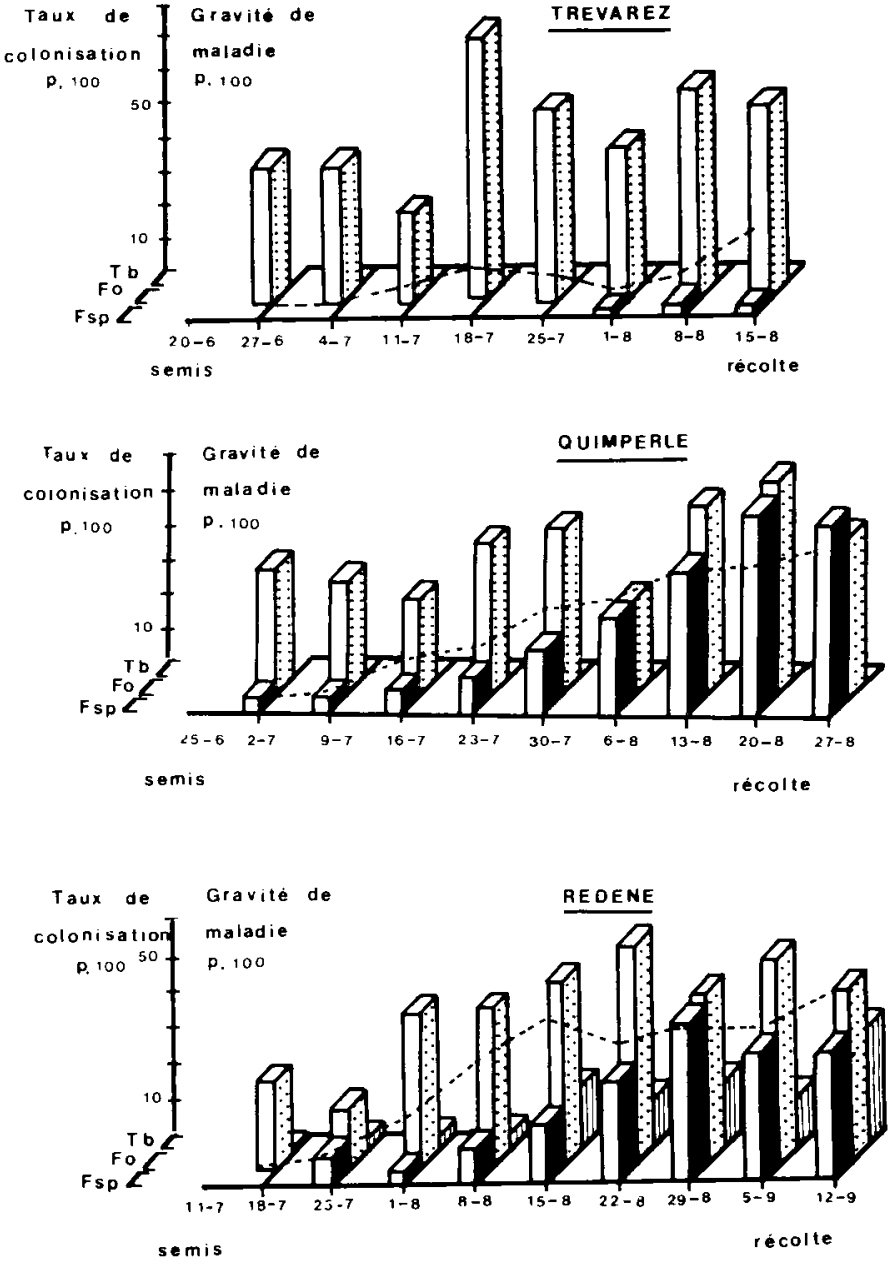

Figure 4

Relations contre lévolution de la gravité de la maladie du pied du haricot (--) au cotws de la culture et la mucoftore présente sur le pied des plantes: F.s. phascoli ( $)$. F. oxysporum ( j, T. basicola ( ). Eude réalisée dans 3 situations géographiques: TREV AREZ, QUIMPERLE, REDENE.

Relationship between the development of hean foot dise'ase (---) during (rop) growth and the plant foot micoflora: F.s. phascoli (口), F. oxysporum, ), T. basicola ) Data from 3 geographic positions : TREVAREZ, QUIMPERLÉ. RÉEENE.

F.s.phaseoli; les populations de T. hasicola évoluent ensuite progressivement, parallèlement à celles de $F$. s. phaseoli et au développement de la maladie.

B. Mise en évidence du rôle de Fusarium solani f. sp. phaseoli et Thielaviopsis basicola dans la maladie

Les résultats, homogènes pour les 4 isolats appartenant à une même espèce fongique, permettent de classer les principaux constituants du complexe en 2 grandes catégories (fig. 5) :

\section{Des champignons non pathogènes sur haricot}

Ce groupe concerne les isolats de $F$. oxysporum qui ne provoquent aucune nécrose (IP inférieur à 17 p. 100 sur le collet, inférieur à 15 p. 100 sur les racines), ce qui confirme les observations de TAYLOR \& PARKINSON (1965). 


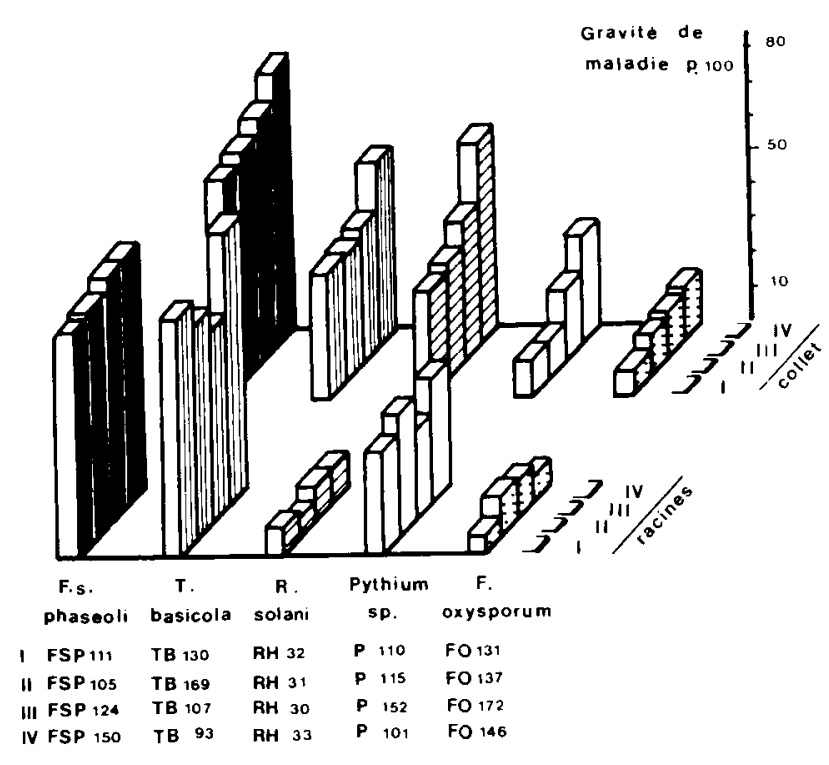

Figure 5

Eade du pouvoir pathogene sur haricot des principanx champignons constituant le complexe parasitaire. Infestation réalisée selon la lédnique "vermiculite" pour F.s. phaseoli, F. oxysporum, R. solani. Pythium sp., et la technique "suspension de spores" pou $\mathrm{T}$. basicola. Les 20 isolats (tabl. 1) sont répartis en 4 groupes $(I, I I, I I, I V$, en fonction de leur agressivite sur le collet des plantes.

Analysis of pathogenicity on French bean of the main fungi involved in the parasitic complex. Inoculation by the "vermiculite" technic for F.s. phaseoli, F. oxysporum, R. solani. Pythium sp., and hy the "spore suspension " technic for T. basicola.

The 20 strains (table 1) were distributed into four groups (I, II, III, IV) according to their aggressiveness on the plant collar.

\section{Des champignons pathogènes sur haricot}

Parmi eux, 2 espèces peu agressives: $R$. solani et Pythium sp; $R$. solani, surtout responsable de fontes de semis, peut néanmoins provoquer au collet des nécroses rouges en forme de tache ovale (IP compris entre 30 et 60 p. 100) ; les racines sont très peu affectées (IP voisin de $10 \mathrm{p} .100)$. Les souches de Pythium également responsables de fontes de semis sont parfois à l'origine de nécroses sur les parties souterraines, mais globalement, les attaques restent faibles; le collet des plantes est généralement peu touché (IP compris entre 10 et 30 p. 100), les nécroses ont plutôt lieu sur racines (IP voisin de 30 p. 100).

Deux espèces sont très agressives: $F$. s. phaseoli et T. basicola (IP supérieurs à $40 \mathrm{p}$. 100). Les nécroses et pourritures du collet ct des racines provoquées par ces 2 champignons sont tout à fait comparables aux symptômes que l'on peut observer au champ. Il est néanmoins possible de les différencier d'après quelquies critères simples: les symptômes dus au Fusarium sont rougeâtres et apparaissent préférenticllement sur le collet des plantes, tandis que ceux dus à $T$. basicola sont brun-noir et importants surtout sur les racines.

\section{DISCUSSION. CONCLUSION}

Les résultats mettent en évidence la diversité de la mycoflore présente sur le pied du haricot et confirment l'hypothèse de l'existence d'un complexe parasitaire responsable de la maladie en France. Parmi les 5 prin- cipales espèces fongiques isolées à partir de plantes malades, 4 sont susceptibles de provoquer des attaques, à des degrés divers:

$R$. solani et Pythium sp., peu fréquents et aussi peu agressifs dans nos conditions expérimentales, semblent jouer un rôle secondaire dans la région étudiée, contrairement à ce qu'ont pu observer d'autres auteurs dans des conditions vraisemblablement différentes: Messiaen \& Laron (1970) dans le Midi de la France; SirRy et al. (1974) en Egypte; Pieczarka \& Abawi (1978) aux Etats-Unis. Par contre, F.s. phaseoli et $T$. basicola, dont les fréquences globalement élevées sont corrélées à l'importance des attaques, sont par ailleurs très agressifs. On peut donc affirmer qu'ils sont les agents responsables directs de la maladie du pied du haricot en Bretagne. Quant aux F. oxysporum, très abondants, nous avons montré leur inocuité directe vis-a-vis du haricot, confirmant ainsi les résultats obtenus par DAvET et al. (1980) au Liban; ces Fusarium peuvent probablement être assimilés aux formes "saprophytes» auxquelles TAYLOR \& PARKINSON (1965) attribuent un fort pouvoir colonisateur du système racinaire du haricot.

Il est intéressant de noter que les 3 principales espèces fongiques observées sur les pieds malades n'apparaissent jamais seules, mais associées par 2 ou par 3 , selon 3 séquences possibles: F. oxysporum-F.s.phaseoli: F. oxysporum-T. basicola; F. oxysporum-F. s. phaseoliT. basicola. En conséquence, la maladie peut être considérée comme la résultante des interactions entre les différentes espèces constituant le complexe parasitaire ; cette conception diffère de celles le plus souvent admises par le passé, qui consistent à ne prendre en compte que l'espèce la plus fréquente.

Les conséquences de cette interprétation se situent à 2 niveaux :

- sur le plan agronomique, l'élaboration et la mise en ouvre de méthodes de lutte contre la maladie du pied sont assujetties à la fragilité de l'équilibre entre les espèces ;

- sur le plan scientifique, le complexe parasitaire constitue un modèle particulièrement intéressant : divers schémas d'interactions entre ses principaux constituants sont en effet envisageables, qui peuvent conduire à des phénomènes d'accroissement ou au contraire de réduction de la maladie; on peut notamment soupçonner une complémentarité entre les 2 espèces pathogènes, T. basicola étant agressif vis-à-vis d'autres plantes telles que le pois et l'épinard, et surtout inféodé aux racines, F.s. phaseoli étant plus spécifique du haricot et plutôt localisé au collet des plantes (LECHAPPE \& RouXEL, 1985). Mais on peut aussi s'interroger sur le rôle du $F$. oxysporum dans l'équilibre du complexe parasitaire et par conséquent dans l'évolution de la maladie : cette espèce omniprésente dans les tissus du pied du haricot pourrait jouer un rôle de précurseur en favorisant la pénétration des agents pathogènes, ou bien accélérer la dégradation des tissus nécrosés comme l'a montré DAveT (1976) dans le cas de la tomate; il est aussi possible que la présence du $F$. oxysporum conduise à une certaine protection de la plante contre les agressions parasitaires.

Par ailleurs, l'examen de la dynamique de la colonisation parasitaire révèle que les différentes espèces 
fongiques s'installent successivement sur la plante; plusieurs hypothèses seraient à vérifier pour tenter d'expliquer ces successions, soit à partir de l'équipement enzymatique de chacun des champignons, soit à partir du degré de réceptivité de la plante en fonction de son stade physiologique; mais on sait également que la température optimale d'agressivité du F.s.phaseoli $\left(25^{\circ} \mathrm{C}\right)$ diffère de celle du T. basicola $\left(17^{\circ} \mathrm{C}\right)$ (LECHAPPE \& RouXeL, 1985). Parmi les paramètres susceptibles d'expliquer la diversité des successions et séquences parasitaires, il convient donc de considérer les facteurs écologiques (climat, sol, environnement), et les pratiques culturales (compactage) dont BURKE \& MiLLER (1983) ont déjà signalé le rôle prépondérant dans l'expression de la maladie due à $F$. s. phaseoli.

Face à ce large faisceau de prolongements possibles, l'étude des interactions entre les 3 principaux champignons associés au niveau du pied du haricot
(F. oxysporum, F. s. phaseoli, T. basicola) nous apparait comme l'un des axes à privilégier; au-delà de son intérêt agronomique lié à l'incidence de l'équilibre du complexe parasitaire sur l'évolution de la maladie, ce modèle doit permettre d'étudier en conditions contrôlées le rôle de divers facteurs (sol, plante, environnement) sur l'équilibre et la dynamique des populations microbiennes.

Reçu le 25 aotit 1987. Accepté le 24 févier 1988.

\section{REMERCIEMENTS}

Cette étude a été réalisée grâce au concours financier de l'Etablissement Public Régional de Bretagne. Les auteurs remercient les différents organismes ayant participé aux travaux sur le terrain : Comité Economique Régional de Bretagne, Union Interprofessionnelle des Légumes de Conserve, G.R.C.E.T.A. de Quimperlé, Chambres d'Agriculture du Finistère et du Morbihan, Service de la Protection des Végctaux, circonscription Bretagne.

\section{RÉFÉRENCES BIBLIOGRAPHIQUES}

Burke D. W., 1965. Fusarium root rot of beans and behaviour of the pathogen in different soils. Phytopathology, 55, 254-256.

Burke D. W., Miller D. E., 1983. Control of Fusarium root rot with resistant beans and cultural management. Plant. Dis. Rép., 67, 1312-1317.

Burkholder W. H., 1919. The dry root rot of the bean. In Papavizas G. C. \& Davey C. B., 1961. Phytopathology, 51, 92-96.

Campbell C. L., Madden L. V., Pennypacker S. P., 1980. Structural characterization of bean root rot epidemics. Phytopathology, 70, 152-155.

Davet P., 1976. Etude des pourritures des racines de la Tomate au Liban ot du complexe parasitaire qui leur est associé. Thèse Docteurès-Sciences Naturelles, Univ. Nancy I, $120 \mathrm{p}$.

Davet P., Ravise A., Baroudy C., 1980. La microflore fongique des racines du Haricot au Liban. Ann. Phytopathol., 12, 235-252.

Decharme M., Coutin R., 1980. Les maladies du Haricot dans " $L e$ Haricot Mange-tout et le Flageolet ». Revue INVUFLEC. $210 \mathrm{p}$.

Gupta V. K., Saharan G. S., 1973. Seed rot and root rot complex of beans (Phaseolus vulgaris L.). Biol. Plant., 15 (2), 123-126.

Lechappe J., Rouxel F., 1985. La Maladie du pied du Haricot.

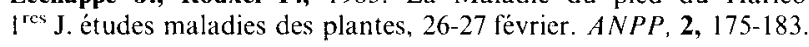

Maloy O. C., 1959. Microbial associations in the Fusarium root rot of beans. Plant. Dis. Rep., 43, 929-933.

Nash S. M., Snyder W. C., 1962. Quantitative estimations by plate counts of propagules of the bean root rot Fusarium in field soils. Phytopathology, 52, 567-572.
Messiaen C. M., Cassini R., 1968. Recherches sur les Fusarioses. IV. La systématique des Fusarium. Amn. Epiphyties, 19, 387-454.

Messiaen C. M., Lafon R., 1970. Les maladies des plantes maraichires, 441 p., $2^{\circ}$ édit., INRA, Paris.

Papavizas G. C., Adams P. B., 1969. Survival of root-infecting fungi in soil. XII Germination and survival of endoconidia and chlamydospores of Thiclaviopsis hasicola in fallow soil and in soil adjacent to germinating bean seed. Phytopathology, 59, 371-378.

Papavizas G. C., Davey C. B., 1961. Isolation of Thielaviopsis basicola from bean rhizosphere. Phytopathology, 51, 92-96.

Pfender W. F., Hagedorn D. J., 1982. A phanomyces root and stem rot of snap bean (Abstr.). Phytopathology, 71, 250.

Pieczarka D. J., Abawi G. S., 1978. Effect of interaction between Fusarium, Pythium, and Rhizoctonia on severity of bean root rot. Phytopathology, 68, 403-408.

Reeleder R. D., 1981. Eflect of Pythium populations on severity of bean root rot in Wisconsin. Phytopathology, 71, 110-116.

Sirry A. R., Higazy M. F. H., Faharat A. A., 1974. Studies on root rot disease of Phaseolus vulgaris L. caused by Rhizoctonia solani Kühn. Agric: Res. Rev., 52, 23-29.

Summer D. R., Johnson A. W., Glaze N. C., Dowler C. C., 1978. Root diseases of snap bean and southern pea in intensive cropping systems. Phytopathology, 68, 955-961.

Taylor G. S., Parkinson D., 1965. Studies on fungi in the root region. IV. Fungi associated with the roots of Phaseolus vulgaris L. Plant Soil, 22, 1-20. 